\title{
Comorbidities increase in-hospital mortality in dengue patients in Brazil
}

\author{
Guilherme L Werneck ${ }^{1 /+}$, Alejandro E Macias ${ }^{2}$, Cesar Mascarenas ${ }^{3}$, Laurent Coudeville ${ }^{3}$, David Morley , \\ Vincent Recamier ${ }^{4}$, Mariana Guergova-Kuras ${ }^{4}$, Esteban Puentes-Rosas ${ }^{5}$, Nicolas Baurin ${ }^{3}$, Myew-Ling Toh ${ }^{3}$
}

\author{
${ }^{1}$ Universidade Federal do Rio de Janeiro, Instituto de Estudos em Saúde Coletiva, Rio de Janeiro, RJ, Brasil \\ ${ }^{2}$ Universidad de Guanajuato, Departamento de Medicina, Área de Microbiología, Guanajuato, Mexico \\ ${ }^{3}$ Sanofi Pasteur, Lyon, France \\ ${ }^{4}$ Ariana Pharmaceuticals, Paris, France \\ ${ }^{5}$ Sanofi Pasteur, Mexico
}

Dengue remains an unmet public health burden. We determined risk factors for dengue in-hospital mortality in Brazil. Of 326,380 hospitalised dengue cases in 9-45-year-old individuals, there were 971 deaths. Risk of dying was 11-times higher in the presence of underlying common comorbidities (renal, infectious, pulmonary disease and diabetes), similar to the risk of dying from severe dengue and much higher with the combination. Ensuring access to integrated dengue preventative measures in individuals aged $\geq 9$ years including those with comorbidities may help achieve the WHO objective of 50\% reduction in mortality and 25\% reduction in morbidity due to dengue by 2020 .

Key words: dengue - haemorrhagic fever - Latin America - case fatality rate

In Brazil, dengue is spreading geographically with increasing numbers of reported cases and severity. Recent surveillance data indicate that between 2010-2016 Brazil suffered an average of 1,077,025 suspected, and 245,925 laboratory-confirmed dengue cases, annually (PAHO 2017). However, the true burden is likely underestimated (Sarti et al. 2016). According to Fares et al. (2015), the incidence of dengue in Brazil has frequently been high, and the number of cases in the country has at some point in time represented up to $60 \%$ of the dengue cases reported worldwide (Fares et al. 2015), with significant economic costs of US\$ 728 million in 2013, mostly due to ambulatory dengue cases (Shepard et al. 2016).

The reported case fatality rate (CFR) is a measure of the severity of a disease and is defined as the proportion of reported cases of a specified disease or condition which are fatal within a specified time (WHO 2018). Dengue death rates remain high and the goal of reducing CFR for dengue haemorrhagic fever to $<1 \%$ set by the World Health Organization (WHO) and the National Dengue Control program in Brazil remains unreached (WHO 1997, MS 2002). In Brazil, the Pan American Health Organization (PAHO) has reported CFRs for dengue cases ranging from 0.04 to $4.07 \%$ annually (PAHO 2017). However, CFRs as high as $18.6 \%$ were reported for dengue patients in intensive care units (ICU) and $19.6 \%$ for in-hospital patients in a recent study in Minas Gerais state in southeastern Brazil (Amancio et al. 2015, Durand et al. 2017). As dengue CFR in Brazil may differ

doi: 10.1590/0074-02760180082

Financial support: Sanofi Pasteur

+ Corresponding author: gwerneck@iesc.ufrj.br

Received 13 February 2018

Accepted 17 May 2018 by two orders of magnitude from year to year, other dengue infection factors must be considered in estimating the disease severity. Comorbidities are highly prevalent in dengue endemic areas including Brazil, and contribute to some of the highest death rates and public health burden in those countries (Mehta and Hotez 2016). Dengue patients with comorbidities may be at higher risk of severe dengue and death, however, there have been no large scale studies conducted in Brazil (Figueiredo et al. 2010, Teixeira et al. 2015, Toledo et al. 2016).

In a large retrospective cohort study, we determined risk factors for dengue in-hospital mortality using the Brazilian Hospital Information System of the Unified Health System (SIH/SUS) database over an eight year period (2008-2015) (Ministério da Saúde - Available from: http://sihd.datasus.gov.br/). Hospital admission records (92 million) from 20,576 hospital departments (e.g. pediatric, clinical) at 5,983 hospitals were included. Patient records from 326,380 hospitalised dengue cases in 9-45-year-old individuals [173,778 female (53.2\%) and $152,602$ male $(46.8 \%)]$ were analysed. This broad age range includes adolescents, young adults, and adults, thereby excluding the elderly who may have a high prevalence of comorbidities and thus limit the ability to detect differences in CFR. Ethical approval was not required as the database was publically accessible. In-hospital mortality was identified with dengue cases and comorbidities identified directly from the recorded principal and secondary diagnoses for each hospitalised dengue case using the International Classification of Diseases, 10th Revision (ICD 10) codes (WHO 2014). Dengue cases were divided into either non-severe $($ ICD 10 code $=$ A90; classical dengue) or severe dengue diagnosis codes (ICD 10 code $=$ A91; dengue haemorrhagic fever).

In order to exclude potential bias in the analysis, as comorbidities may also be a consequence of severe dengue manifestations, codes considered as severe dengue symptoms and complications were excluded. The comor- 
bidity definitions used are summarised in Supplementary data, Table. Analyses were performed using the KNIME (Berthold et al. 2008) analytic platform integrated with $\mathrm{KEM}^{\circledR}$ (Ariana Pharmaceuticals; Liquiere and Sallantin 1998) data mining tools, MySQL database and R v3.0.3 statistical software. Data were presented as CFRs and relative mortality ratios (RMR) and $95 \%$ confidence intervals (CI) compared to different groups. P values for between group RMRs were determined for statistical significance by Fisher's exact test, whereby $p<0.05$ was considered statistically significant.

We first determined whether mortality rates were increased in hospitalised dengue patients with underlying comorbidities compared to those without comorbidities. Of the 326,380 hospitalised dengue cases in 9-45-year-old individuals there were 971 deaths resulting in an overall CFR of $0.30 \%$ in this age group (Table). The crude RMR from hospitalised dengue was 11-times higher (95\% CI 9-15) in the presence of common comorbidities (CFR 3.07\%, $\mathrm{p}<0.001$ ) compared to the absence of comorbidities (CFR $0.27 \%$ ).

The risk of dying from hospitalised dengue in those with non-severe dengue and underlying comorbidities (CFR 1.94\%, RMR 13, 95\% CI 9-20, p < 0.001) was not statistically different to the risk of dying from severe dengue haemorrhagic fever with no underlying comorbidity (CFR 2.73\%, RMR 19, 95\% CI 17-22, p < 0.001) (Table). The risk of dying was further significantly increased with the combination of severe dengue haemorrhagic fever and underlying comorbidities (CFR 15.33\%, RMR $106,95 \%$ CI $71-159, \mathrm{p}<0.001)$ compared to individuals with dengue alone (non-severe dengue and no underlying comorbidity). Furthermore, there was a 1.7fold higher prevalence of severe dengue associated with comorbidities $(8.4 \%, \mathrm{p}<0.001)$, compared to without comorbidities $(4.8 \%)$ (Table).

Thus, the presence of both comorbidities and severe dengue are cumulative risk factors for increased hospitalised dengue death rates. Comorbidities with highest dengue hospital death rates, regardless of dengue severity, were consistently renal disease, infectious disease, pulmonary disease and diabetes. However, in the presence of both severe dengue and underlying comorbidities, such as diabetes (CFR 25\%), other non-dengue infectious diseases (CFR 20.83\%), pulmonary disorders (CFR 20.59\%), renal disease (CFR 15.38\%); fatality rates were higher than in individuals with non-severe dengue and no underlying comorbidities (Table).

To our knowledge, this is the largest retrospective cohort study of more than 300,000 hospitalised dengue cases $(15,993$ dengue haemorrhagic fever, 971 fatal cases) demonstrating a high mortality in the presence of comorbidities in individuals 9-45 years of age in Brazil over an eight year period. Our study demonstrated for the first time that risk of dengue death in the presence of comorbidities is similar to the risk of death from severe dengue and much higher with the combination of both comorbidities and severe dengue. The comorbidities contributing to increased death rates were consistently infectious diseases, pulmonary diseases, renal disease/failure and diabetes regardless of dengue severity.
Prior publications on the impact of underlying comorbidities on dengue related mortality are limited and inconsistent, in part related to the small number of deaths included in these analyses (Toledo et al. 2016). However, these reports suggest that underlying comorbidities can worsen dengue clinical outcomes.

Two large scale studies explored severe dengue as a risk factor for dengue mortality but did not specifically analyse the contribution of comorbidities. Both studies used the Brazilian Information System for Notifiable Diseases (SINAN) and gathered information on 281,159 and 105,459 dengue cases and 156 and 62 deaths, respectively (Campos et al. 2015, Pinto et al. 2016). Campos et al. (2015) reported that factors associated with dengue mortality included age $>65$ years and plasma leakage. Pinto et al. (2016) found that patients who died due to severe dengue had more hematuria, gastrointestinal bleeding, and thrombocytopenia than survivors. In Brazil, there have been two further publications on a small series of patients. In a case-controlled study with 1345 patients during 20032005 ( $\mathrm{n}=170$ dengue haemorrhagic fever cases), diabetes (OR 2.75; 95\% CI 1.12-6.73) was associated with dengue haemorrhagic fever (Figueiredo et al. 2010). In a later casecontrolled study with 1806 patients during 2009-2012, hypertension (OR 1.6; 95\% CI 1.1-2.1) and skin allergy (OR 1.8; 95\% CI 1.1-3.2) increased the likelihood of progressing to dengue haemorrhagic fever but diabetes was not a confirmed risk factor (Teixeira et al. 2015). A study of the 1981 dengue epidemic in Cuba found that chronic diseases such as bronchial asthma, diabetes mellitus and sickle cell anaemia were more frequently observed in fatal dengue cases (Bravo et al. 1987). A number of other studies have also suggested that underlying comorbid conditions are common among dengue-related deaths, but these have generally been based on analyses that included very few deaths (typically, $<15$ deaths included in the analyses) (Guzman et al. 1999, Lee et al. 2005, Ong et al. 2007, Lahiri et al. 2008, Lee et al. 2008). The estimated CFR of $0.3 \%$ in all hospitalised dengue cases in our study was much lower than the $1.3 \%$ average reported to PAHO for Brazil during the same time period, but this may be due to differences in reporting of severe dengue, which may elevate the CFR in other studies. Furthermore, this may reflect the inherent differences in clinical case management practices and dengue diagnosis for dengue/severe dengue (Kalayanarooj 1999), or differences in circulating dengue virus serotypes, which all co-circulate but vary unpredictably over time (Ocazionez et al. 2006, OhAinle et al. 2011). In our study, although dengue diagnosis was based on a combination of clinical diagnosis and/or laboratory diagnosis, this reflects current clinical practice guidelines by the WHO in countries where laboratory diagnosis may not be available. We have also conducted a more in-depth analysis of the individual risk factors and determined that age, severe dengue and comorbidities are independent and cumulative risk factors for increased dengue mortality in Brazil and other countries in Latin America (submitted for publication). Despite the fact that we excluded all severe dengue symptoms and pathologies associated with severe dengue outcome, it is possible that there remains still some underlying bias whereby increased risk of death 


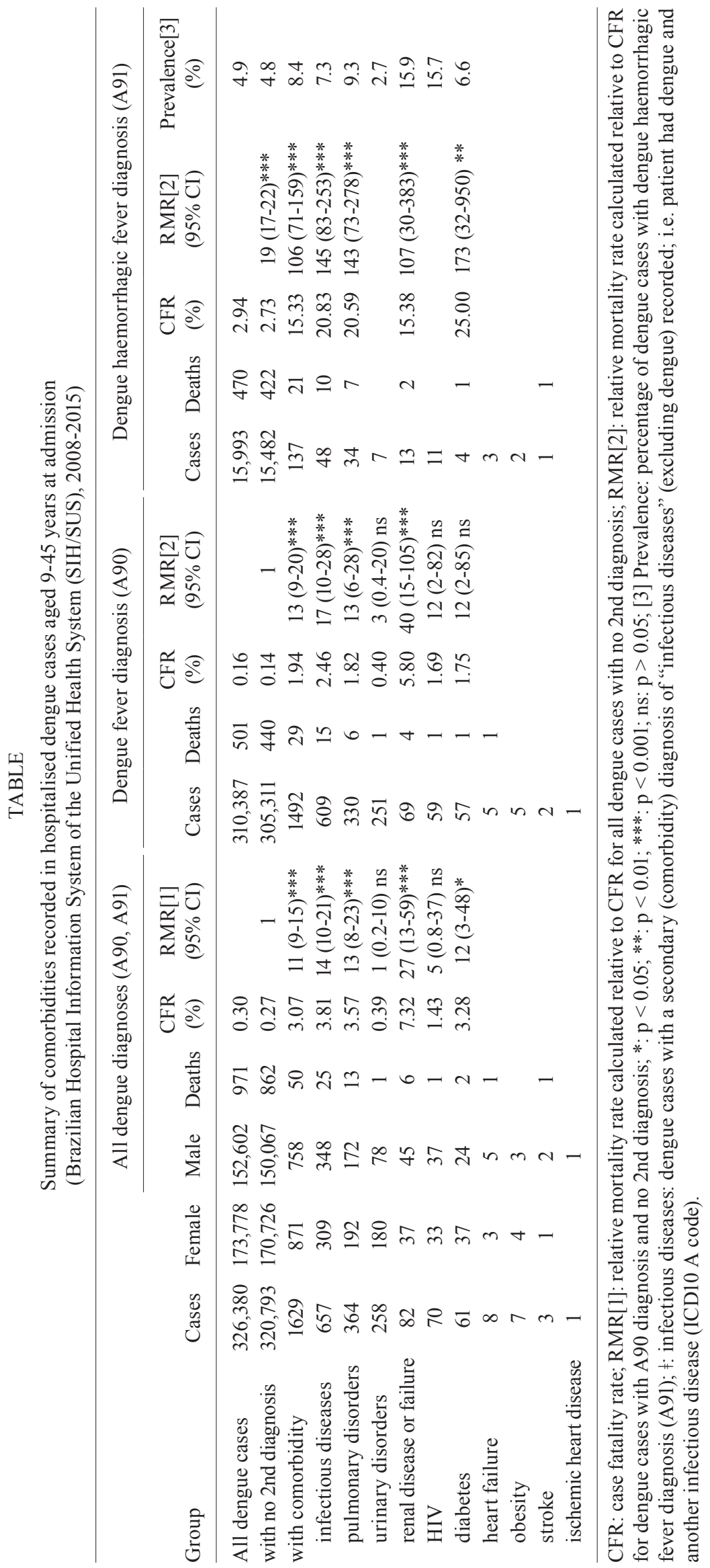


from comorbidities may be just a manifestation of severe dengue induced comorbidities. For example, we considered renal disease or failure as a comorbid condition, but acute renal failure may be a complication of severe dengue (Wiwanitkit 2005). However, overall, the data on renal disease also included chronic renal failure and other underlying renal pathologies which are not outcomes of severe dengue. Socioeconomic and healthcare factors may also play a role in dengue mortality; in Brazil, dengue mortality was shown to be associated with a number of socioeconomic and urbanisation indicators (Paixão et al. 2015). Nonetheless, a major strength of our study is the large number of deaths assessed in our analysis, which encompasses reported cases across the whole of Brazil. Further, our study used the SIH/SUS reporting system which is unique in allowing the analysis of comorbidities in a detailed way.

In conclusion, the risk of dying from hospitalised dengue is 11-times higher in those with underlying common comorbidities. To our knowledge this is the largest retrospective analysis conducted in Brazil. The comorbidities identified in a high proportion of dengue deaths were renal disease, infectious disease, pulmonary disease and diabetes. Ensuring access to dengue preventative measures (e.g., dengue vaccine, vector control and prompt medical care) in individuals with comorbidities may help achieve the WHO objective of 50\% reduction in dengue-related mortality and $25 \%$ reduction in morbidity by 2020 (WHO 2012).

\section{ACKNOWLEDGEMENTS}

To Jean-Sébastien Persico for editorial assistance with the coordination of this manuscript on behalf of Sanofi Pasteur.

\section{AUTHORS' CONTRIBUTION}

All authors were involved in the concept and design of the study. All authors contributed to the interpretation of the data and participated in the preparation of this manuscript, and approved the final manuscript for submission. All authors had access to the study data and are responsible for the veracity and completeness of the data reported. Conflict of interest: AEM has no conflicts of interest. GW was partially funded by Sanofi Pasteur; CM, LC, EP-R, NB and M-LT are employees of Sanofi Pasteur; DM, VR and MG-K are employees of Ariana Pharmaceuticals who were contracted to undertake the analysis.

\section{REFERENCES}

Amancio FF, Heringer TP, de Oliveira CC, Fassy LB, de Carvalho FB, Oliveira DP, et al. Clinical profiles and factors associated with death in adults with dengue admitted to intensive care units, Minas Gerais, Brazil. PLoS One. 2015; 10(6): e0129046.

Berthold MR, Cebron N, Dill F, Gabriel TK, Meinl T, Ohl P, et al. KNIME: the konstanz information miner. In: Preisach C, Burkhardt H, Schmidt-Thieme L, Decker R, edotors. Data analysis, machine learning and applications. Studies in classification, data analysis, and knowledge organization. Springer, Berlin, Heidelberg; 2008.

Bravo JR, Guzman MG, Kouri GP. Why dengue haemorrhagic fever in Cuba? Individual risk factors for dengue haemorrhagic fever/ dengue shock syndrome (DHF/DSS). Trans R Soc Trop Med Hyg. 1987; 81(5): 816-20.

Campos KB, Amancio FF, de Araujo VE, Carneiro M. Factors associated with death from dengue in the state of Minas Gerais, Brazil: historical cohort study. Trop Med Int Health. 2015; 20(2): 211-8.
Durand L, Genovez V, Araujo D. Cost effectiveness of dengue vaccination in Brazil/Custo-efetividade da vacinação contra dengue no Brasil. J Bras Econ Saude. 2017; 9(1): 12-29.

Fares RC, Souza KP, Anez G, Rios M. Epidemiological scenario of dengue in Brazil. Biomed Res Int. 2015; 2015: 321873.

Figueiredo MA, Rodrigues LC, Barreto ML, Lima JW, Costa MC, Morato V, et al. Allergies and diabetes as risk factors for dengue hemorrhagic fever: results of a case control study. PLoS Negl Trop Dis. 2010; 4(6): e699.

Guzman MG, Alvarez M, Rodriguez R, Rosario D, Vazquez S, Vald SL, et al. Fatal dengue hemorrhagic fever in Cuba, 1997. Int J Infect Dis. 1999; 3(3): 130-5.

Kalayanarooj S. Standardized clinical management: evidence of reduction of dengue haemorrhagic fever case-fatality rate in Thailand. Dengue Bulletin. 1999; 23: 10-17.

Lahiri M, Fisher D, Tambyah PA. Dengue mortality: reassessing the risks in transition countries. Trans R Soc Trop Med Hyg. 2008; 102(10): 1011-16

Lee IK, Liu JW, Yang KD. Clinical and laboratory characteristics and risk factors for fatality in elderly patients with dengue hemorrhagic fever. Am J Trop Med Hyg. 2008; 79(2): 149-53.

Lee IK, Liu JW, Yang KD. Clinical characteristics and risk factors for concurrent bacteremia in adults with dengue hemorrhagic fever. Am J Trop Med Hyg. 2005; 72(2): 221-6.

Liquiere M, Sallantin J. Structural machine learning with galois lattice and graphs. In: ICML'98: 5th International Conference on Machine Learning. Madison: 1998. 305-13.

Mehta P, Hotez PJ. NTD and NCD Co-morbidities: the example of dengue fever. PLoS Negl Trop Dis. 2016; 10(8): e0004619.

MS - Ministério da Saúde. Fundação Nacional de Saúde. Programa Nacional de Controle da Dengue - PNCD, FUNASA. Brasília: MS; 2002. Available from: http://bvsms.saude.gov.br/bvs/publicacoes/pncd_2002.pdf.

Ocazionez RE, Cortes FM, Villar LA, Gomez SY. Temporal distribution of dengue virus serotypes in Colombian endemic area and dengue incidence. Re-introduction of dengue- 3 associated to mild febrile illness and primary infection. Mem Inst Oswaldo Cruz. 2006; 101(7): 725-31.

OhAinle M, Balmaseda A, Macalalad AR, Tellez Y, Zody MC, Saborio $\mathrm{S}$, et al. Dynamics of dengue disease severity determined by the interplay between viral genetics and serotype-specific immunity. Sci Transl Med. 2011; 3(114): 114ra128.

Ong A, Sandar M, Chen MI, Sin LY. Fatal dengue hemorrhagic fever in adults during a dengue epidemic in Singapore. Int J Infect Dis. 2007; 11(3): 263-7.

PAHO - Pan American Health Organization. Dengue: data, maps and statistics. 2017. Available from: http://www.paho.org/hq/index. php?option=com_topics\&view $=$ article $\&$ id $=1 \&$ Itemid $=40734$.

Paixão ES, Costa MC, Rodrigues LC, Rasella D, Cardim LL, Brasileiro $\mathrm{AC}$, et al. Trends and factors associated with dengue mortality and fatality in Brazil. Rev Soc Bras Med Trop. 2015; 48(4): 399-405.

Pinto RC, Castro DB, Albuquerque BC, Sampaio VS, Passos RA, Costa $\mathrm{CF}$, et al. Mortality predictors in patients with severe dengue in the state of Amazonas, Brazil. PLoS One. 2016; 11(8): e0161884.

Sarti E, L’Azou M, Mercado M, Kuri P, Siqueira JB, Solis E, et al. A comparative study on active and passive epidemiological surveillance for dengue in five countries of Latin America. Int J Infect Dis. 2016; 44: 44-9. 
Shepard DS, Undurraga EA, Halasa YA, Stanaway JD. The global economic burden of dengue: a systematic analysis. Lancet Infect Dis. 2016; 16(8): 935-41.

Teixeira MG, Paixão ES, Costa MC, Cunha RV, Pamplona L, Dias JP, et al. Arterial hypertension and skin allergy are risk factors for progression from dengue to dengue hemorrhagic fever: a case control study. PLoS Negl Trop Dis. 2015; 9(5): e0003812.

Toledo J, George L, Martinez E, Lazaro A, Han WW, Coelho GE, et al. Relevance of Non-communicable comorbidities for the development of the severe forms of dengue: a systematic literature Review. PLoS Negl Trop Dis. 2016; 10(1): e0004284.

WHO - World Health Organization. Dengue haemorrhagic fever: diagnosis, treatment, prevention and control. 2nd ed. Geneva:
World Health Organization. 1997. Available from: http://apps. who.int/iris/bitstream/10665/41988/1/9241545003_eng.pdf.

WHO - World Health Organization. Global health observatory (GHO) data. 2018. Available from: http://www.who.int/gho/epidemic diseases/cholera/case_fatality_rate_text/en/.

WHO - World Health Organization. Global strategy for dengue prevention and control, 2012-2020. 2012. Available from: http://apps.who. int/iris/bitstream/10665/75303/1/9789241504034_eng.pdf?ua=1.

WHO - World Health Organization. World Health Organization International Statistical Classification of Diseases and Related Health Problems 10th Revision. 2014 Available from: http://apps.who. int/classifications/icd10/browse/2014/en\#/II.

Wiwanitkit $\mathrm{V}$. Acute renal failure in the fatal cases of dengue hemorrhagic fever, a summary in Thai death cases. Ren Fail. 2005; 27(5): 647. 